\title{
Time Evolution of Various Cosmological Parameters and Their Inter-Dependence in the Framework of Brans-Dicke Theory
}

\author{
Sudipto Roy \\ Department of Physics, St. Xavier's College, Kolkata, 30 Mother Teresa Sarani (Park Street), \\ Kolkata-700016, West Bengal, India. email: roy.sudipto1@gmail.com
}

\begin{abstract}
A theoretical model, regarding the time dependence of several cosmological parameters, has been constructed in the framework of generalized Brans-Dicke theory, using its field equations for spatially flat FRW metric. An empirical expression of the scalar field parameter, written in terms of the scale factor, has been substituted into the field equations to obtain expressions of Brans-Dicke dimensionless parameter $(\omega)$ and the density of matter $(\rho)$, in terms of several relevant cosmological parameters. The dark energy, which is regarded as responsible for the accelerated expansion of the universe, is assumed to have generated from matter. On the basis of a model of matter to dark energy conversion, an expression of the density of dark energy has been derived. Using these relations of matter and dark energy density, expressions regarding the proportions of matter and dark energy have been formulated. To study the time dependence of all these quantities, an empirical time dependent expression of scale factor has been incorporated into their expressions. This scale factor expression has been so chosen that, the deceleration parameter derived from it, changes sign from positive to negative, as time goes on, implying the widely accepted change of phase from deceleration to acceleration, in cosmic expansion. Time dependence of gravitational constant and various other parameters and their interdependence have been depicted graphically.
\end{abstract}

Keywords: Densities of matter and dark energy, Time dependence of Brans-Dicke parameter and scalar field, Relative proportions of matter and dark energy, Dependence of cosmic expansion upon matter content, Time dependence of gravitational constant, Cosmology.

\section{Introduction}

On the basis of recent cosmological observations and theoretical findings, based on an extensive analysis of data from supernova, it is now a widely accepted fact, that the universe has made a transition from a phase of decelerated expansion to its present phase of accelerated expansion [1,2], indicated by a negative value of the deceleration parameter. This expansion of the universe was initially believed to be governed solely by gravitational attraction among celestial bodies, which is capable of causing only decelerated expansion. The observation of accelerated expansion of the universe, evident from the negative value of the experimentally determined deceleration parameter, triggered speculations about the existence of a special kind of matter or energy responsible for this acceleration. Interactions of normal matter with this new form of matter/energy are believed to make the effective pressure sufficiently negative, leading to a repulsive effect. Dark energy is the name of this new matter or energy that causes accelerated expansion. A great amount of research has gone into the determination of its true nature. A number of theoretical models have been proposed to explain the findings regarding the accelerated expansion of the universe.

The cosmological constant ( $\Lambda$ ), a well known parameter in the general theory of relativity, has been chosen to represent the entity named dark energy in many models [3]. Models regarding cold dark matter (CDM) have a serious drawback in connection to the value of cosmological constant. The currently observed value of cosmological constant for an accelerating universe does not match with that of the value in Planck scale or Electroweak scale [4]. The problem can be rendered less acute if one tries to construct dark energy models with a time dependent cosmological parameter. Researchers have come across some shortcomings of these models [5].

In order to formulate alternative theories to the dynamical models involving the cosmological constant, the scalar field models were proposed. These are the ones in which the equation of state of dark energy changes with time. Quintessence models, among the many proposed scalar field models, are the ones endowed with a potential so that the contribution to the pressure sector, can evolve to attain an adequately large negative value, thus generating the observed cosmic acceleration [6]. One of the main shortcomings of these quintessence models is that most of the quintessence potentials are chosen arbitrarily and do not have a proper theoretical justification explaining their genesis. Consequently a large number of other alternative scalar field models have been proposed, such as the tachyon [7], k-essence [8], holographic [9] dark energy models in the scientific literature, with their own limitations. 
In most of the scalar field models, the cold dark matter and dark energy are normally allowed to evolve independently. However, there are attempts to include an interaction amongst them so that one grows at the expense of the other [10]. Non minimal coupling of the scalar field with the dark matter sector through an interference term in the action has helped in explaining the cosmic acceleration. One refers to these fields as Chameleon fields and they have successfully represented the dark energy to account for the observations [11].

Non minimal coupling between the scalar field and geometry, based on the structure of the BransDicke (BD) theory of cosmology, can be shown to account for the accelerated expansion of the universe. A potential function term $V(\varphi)$, which is a function of the BD scalar field $\varphi$ itself, is incorporated in a modified form of the BD theory. In explaining the acceleration of the universe, this new model can serve as a strong candidate [12].

In the framework of the BD theory of cosmology, a number of theoretical models have been analyzed and compared with one another. For example, Sheykhi et al. worked with the power-law entropy-corrected version of BD theory defined by a scalar field and a coupling function [13]. Jamil et. al. studied the cosmic evolution in Brans-Dicke chameleon cosmology [14]. Pasqua and Khomenko studied the interacting logarithmic entropy-corrected HDE model in BD cosmology with IR cut-off given by the average radius of the Ricci scalar curvature [15].

As introduced in some models based on the BD theory, a quintessence scalar field can generate a signature flip of the deceleration parameter from positive to negative for a wide range of potentials [16]. An interaction between dark matter and the BD scalar field showed that the matter dominated era can have a transition from a decelerated to an accelerated expansion without any additional potential [17]. The BD scalar field, on the other hand, is alone capable of driving the acceleration without any quintessence matter or without considering any interaction between dark matter and the BD scalar field [18].

Several such models are found to have some discrepancies in the sense that the matter dominated universe has always been expanding with acceleration, which is in contrary to the recent observations in this regard. Apart from this, one has to take a wide range of values of the BD parameter $\omega$ to explain different phenomena. In order to explain the recent acceleration many of the models require a very low value of the $\mathrm{BD}$ parameter $\omega$, of the order of unity, but one needs a very high value of $\omega$ to explain local astronomical experiments [19].

The present study is on the basis of generalized Brans-Dicke theory where the Brans-Dicke dimensionless parameter $(\omega)$ is regarded as a function of the scalar field $(\varphi)$, instead of being treated as a constant. Here, an empirical power law relation, between the scalar field $(\varphi)$ and the scale factor $(a)$, has been used, as has been done in many other studies [20, 21, 26]. It leads to expressions of the density of matter $(\rho)$ and the Brans-Dicke parameter $(\omega)$ in terms of other cosmological parameters such as Hubble parameter, deceleration parameter etc. An expression of the density of dark energy $\left(\rho_{d}\right)$ has been formulated on the basis of an assumption that the entire dark energy content of the universe has been produced from matter. Its value depends on the time at which this conversion process started. Using these expressions of densities of matter and dark energy, expressions regarding the proportions of matter and dark energy have been derived. The values of these relative proportions of matter and dark energy depend, among other parameters, on the time $\left(t=\gamma t_{0}\right)$ at which the process of conversion of matter into dark energy started. The Brans-Dicke parameter $(\omega)$ has been shown to be a function of the scalar field, which is obvious for the generalized Brans-Dicke theory [20]. To derive expressions regarding the time variation of different quantities, a time dependent empirical expression of the scale factor has been used. It has been so chosen that the deceleration parameter, calculated on its basis, changes sign from positive to negative, clearly being consistent with the widely accepted fact that the universe has changed its phase of expansion from deceleration to acceleration. Time dependence of densities and relative proportions of matter and dark energy has been determined. Expressions representing the time dependence of matter and dark energy contents of the universe have been derived. Time dependence of various cosmological parameters has been shown graphically. The effect of depletion of matter content upon the process of cosmic expansion has also been explored graphically.

\section{Field Equations and the Theoretical Model}

In the generalized Brans-Dicke theory, the action is expressed as [22],

$$
S=\int d^{4} x \sqrt{-g}\left(\frac{\varphi R}{16 \pi G}+\frac{\omega(\varphi)}{\varphi} g^{\mu \nu} \partial_{\mu} \varphi \partial_{v} \varphi+L_{m}\right)
$$

Here, $g$ is the determinant of the tensor metric $g^{\mu \nu}, R$ is the Ricci scalar, $L_{m}$ is the Lagrangian of matter, $\varphi$ is the Brans-Dicke scalar field and $\omega$ is a dimensionless Brans-Dicke parameter. Here $\omega$ is considered to be a function of $\varphi$, instead of being regarded as a constant. 
The line element for a non-flat universe, in the framework of the Friedmann-Robertson-Walker (FRW) cosmology, can be expressed in polar coordinates as,

$d s^{2}=-d t^{2}+a^{2}(t)\left[\frac{d r^{2}}{1-k r^{2}}+r^{2} d \theta^{2}+r^{2} \sin ^{2} \theta d \xi^{2}\right]$

Here $a(t)$ is the scale factor of the universe, $t$ represents the cosmic time, $r$ is the radial component, $k$ is the curvature parameter and $\theta$ and $\xi$ are the two polar coordinates.

For a flat FRW universe (which corresponds to the case of $k=0$ ), the field equations in the generalized $\mathrm{BD}$ theory are given by [20].

$3\left(\frac{\dot{a}}{a}\right)^{2}=\frac{\rho}{\varphi}+\frac{\omega(\varphi)}{2}\left(\frac{\dot{\varphi}}{\varphi}\right)^{2}-3 \frac{\dot{a}}{a} \frac{\dot{\varphi}}{\varphi}$

$2 \frac{\ddot{a}}{a}+\left(\frac{\dot{a}}{a}\right)^{2}=-\frac{\omega(\varphi)}{2}\left(\frac{\dot{\varphi}}{\varphi}\right)^{2}-2 \frac{\dot{a}}{a} \frac{\dot{\varphi}}{\varphi}-\frac{\ddot{\varphi}}{\varphi}$

Combining equation (3) with (4) one gets,

$2 \frac{\ddot{a}}{a}+4\left(\frac{\dot{a}}{a}\right)^{2}=\frac{\rho}{\varphi}-5 \frac{\dot{a}}{a} \frac{\dot{\varphi}}{\varphi}-\frac{\ddot{\varphi}}{\varphi}$

From equation (4), the following expression of $\omega(\varphi)$ is obtained.

$\omega(\varphi)=2\left[-2 \frac{\dot{a}}{a} \frac{\dot{\varphi}}{\varphi}-\frac{\ddot{\varphi}}{\varphi}-2 \frac{\ddot{a}}{a}-\left(\frac{\dot{a}}{a}\right)^{2}\right]\left(\frac{\dot{\varphi}}{\varphi}\right)^{-2}$

In the present study we have considered the following empirical expressions of $\varphi[20,26]$.

$\varphi=\varphi_{0}\left(\frac{a}{a_{0}}\right)^{k}=\varphi_{0} a^{k} \quad$ (taking $\left.a_{0}=1\right)$

Combining equation (7) with (5) one gets,

$(2+k) \frac{\ddot{a}}{a}+\left(k^{2}+4 k+4\right)\left(\frac{\dot{a}}{a}\right)^{2}=\frac{\rho}{\varphi_{0} a^{k}}$

Using the standard expressions of the Hubble parameter and the deceleration parameter, which are $H=\frac{\dot{a}}{a}$ and $q=-\frac{\ddot{a} a}{\dot{a}^{2}}$ respectively, equation (8) can be written as,

$k^{2}+k(4-q)+4-2 q-\frac{1}{H^{2}} \frac{\rho}{\varphi}=0$

Using equation (9), one obtains the following expression of the density of matter.

$\rho=\varphi H^{2}\left[k^{2}+(4-q) k+(4-2 q)\right]$

If it is assumed that the dark energy began forming from matter (dark + baryonic) in the past at the time $t=\gamma t_{0}$, where $\gamma<1$, the density of dark energy would be written as,

$\rho_{d}=\frac{\left(\rho a^{3}\right)_{t=\gamma t_{0}}-\rho a^{3}}{a^{3}}$

Thus, the proportion of matter $\left(R_{m}\right)$ and the proportion of dark energy $\left(R_{d}\right)$ in the universe are expressed by the following two equations.

$R_{m}=\frac{\rho a^{3}}{\left(\rho a^{3}\right)_{t=\gamma t_{0}}}$
$R_{d}=\frac{\left(\rho a^{3}\right)_{t=\gamma t_{0}}-\rho a^{3}}{\left(\rho a^{3}\right)_{t=\gamma t_{0}}}$ 
Combining equation (7) with (6) one gets,

$$
\omega(\varphi)=2(2 q-1) \frac{1}{k^{2}}+2(q-1) \frac{1}{k}-2
$$

Now, using equation (7), one may eliminate the parameter $k$ from equation (14) to get the following expression of $\omega(\varphi)$.

$\omega(\varphi)=2(2 q-1)\left[\frac{\ln \left(\varphi / \varphi_{0}\right)}{\ln a}\right]^{-2}+2(q-1)\left[\frac{\ln \left(\varphi / \varphi_{0}\right)}{\ln a}\right]^{-1}-2$

According to the Brans-Dicke theory, the constant $\varphi_{0}$ in equation (15) is $1 / G_{0}$ where $G_{0}$ denotes the present value of gravitational constant. $G_{0}=6.673 \times 10^{-11} \mathrm{~N} \mathrm{~m}^{2} \mathrm{~kg}^{-2}$.

Equation (15) represents an expression of $\omega(\varphi)$ in terms of $\varphi$ and other parameters which are connected to $\varphi$ through equation (7).

To determine the time variations of the quantities discussed above, we have to use a time dependent expression of a scale factor $a(t)$. It is logical to choose a scale factor that generates a signature flip of the deceleration parameter $(q)$ with time, as found in several studies $[21,23,24,26]$. This change of sign of the deceleration parameter, from positive to negative, indicates a transition from a phase of decelerated expansion to accelerated expansion of the universe. Following some studies in this regard [21, 24, 26], we have chosen for this purpose an empirical scale factor, expressed by the following equation.

$a(t)=a_{0}\left(t / t_{0}\right)^{\alpha} \operatorname{Exp}\left[\beta\left(t-t_{0}\right)\right]$

Here, the constants $\alpha, \beta>0$ to make sure that the scale factor increases with time. For this scale factor, the Hubble parameter and the deceleration parameter are respectively given by the following equations,

$H=\frac{\dot{a}}{a}=\beta+\frac{\alpha}{t}$

$q=-\frac{\ddot{a} a}{\dot{a}^{2}}=-1+\frac{\alpha}{(\alpha+\beta t)^{2}}$

Here we find that, for $0<\alpha<1$, we get $q>0$ at $t=0$

As $t \rightarrow \infty$, we have $q \rightarrow-1$. Thus, $q$ changes sign as a function of time, as desirable for the present study.

The values of constant parameters $(\alpha, \beta)$ have been determined from the following conditions.

Condition - 1: $\quad H=H_{0}$ at $t=t_{0}$

Condition-2: $\quad q=q_{0} \quad$ at $t=t_{0}$

The values of $\alpha, \beta$ are thus found to be,

$\alpha=\left(1+q_{0}\right)\left(H_{0} t_{0}\right)^{2}=4.76 \times 10^{-01}$

$\beta=\frac{H_{0} t_{0}-\alpha}{t_{0}}=\frac{H_{0} t_{0}-\left(1+q_{0}\right)\left(H_{0} t_{0}\right)^{2}}{t_{0}}=1.25 \times 10^{-18}$

The values of different cosmological parameters used in the present study are,

$H_{0}=72\left(\frac{K m}{\mathrm{Sec}}\right)$ per Mega Parsec $=2.33 \times 10^{-18} \mathrm{sec}^{-1}{ }^{\prime} t_{0}=14$ billion years $=4.415 \times 10^{17} \mathrm{sec}$

$\varphi_{0}=\frac{1}{G_{0}}=1.498 \times 10^{10} \mathrm{~m}^{-3} \mathrm{Kgs}^{2}, \rho_{0}=2.831 \times 10^{-27} \mathrm{Kg} / \mathrm{m}^{3}$ (dark+baryonic) and $q_{0}=-0.75$.

Combining equation (7) with (16) we get the following time dependence of $\varphi$.

$\varphi=\varphi_{0}\left(t / t_{0}\right)^{\alpha k} \operatorname{Exp}\left[\beta k\left(t-t_{0}\right)\right]$

In Brans-Dicke theory, the gravitational constant $G=1 / \varphi$. Thus we have,

$G=1 / \varphi=1 / \varphi_{0}\left(t / t_{0}\right)^{-\alpha k} \operatorname{Exp}\left[-\beta k\left(t-t_{0}\right)\right]$ or, one may write,

$G=G_{0}\left(t / t_{0}\right)^{-\alpha k} \operatorname{Exp}\left[-\beta k\left(t-t_{0}\right)\right]$

Using equation (24), the fractional rate of change of the gravitational constant is expressed as,

$\frac{\dot{G}}{G}=-k\left(\frac{\alpha}{t}+\beta\right)$

To determine the values of the constant $k$ we need to solve equation (9), which is quadratic in $k$.

Writing the values of all parameters at $t=t_{0}$, this equation becomes,

$k^{2}+k\left(4-q_{0}\right)+4-2 q_{0}-\frac{1}{H_{0}{ }^{2}} \frac{\rho_{0}}{\varphi_{0}}=0$

The roots of the quadratic equation (26) are given by, 
$k_{ \pm}=\frac{1}{2}\left(q_{0}-4 \pm \sqrt{q_{0}^{2}+4 \rho_{0} / \varphi_{0}{H_{0}}^{2}}\right)$

Using the values of the constants, we get from equation (27), $k_{+}=-1.956$ and $k_{-}=-2.794$.

The density of matter $(\rho)$, as expressed by equations (28A, B), is found to be negative for $k=k_{+}$over a certain range of time $(t)$. Moreover, for $k=k_{+}$, the value of $\omega(t)$, as expressed by equation (34), becomes more negative with time, which is not consistent with other studies $[23,26]$. Therefore, for the present analysis, we have chosen $k=k_{-}$.

Substituting from the equations (17), (18) and (23) into equation (10) one gets the following time dependent form of the density of matter $(\rho)$.

$\rho(t)=\frac{e^{\beta k(t-t 0)}(2+k) \varphi_{0}\left[\alpha\{-1+\alpha(3+k)\}+2 \alpha \beta(3+k) t+\beta^{2}(3+k) t^{2}\right]\left(\frac{t}{t_{0}}\right)^{\alpha k}}{t^{2}}$

Combining equation (16) with (28A) one obtains,

$\rho(t)=\left[\frac{a(t)}{a_{0}}\right]^{k}(2+k) \varphi_{0} \frac{(3+k)(\alpha+\beta t)^{2}-\alpha}{t^{2}}$

Using this expression of $\rho(t)$ of equation (28B), assuming $a_{0}=1$, the time dependence of the density of dark energy $\left(\rho_{d}\right)$, following equation (11), is expressed as,

$\rho_{d}=\frac{(2+k) \varphi_{0}}{[a(t)]^{3}}\left[\left[a\left(\gamma t_{0}\right)\right]^{\epsilon} \frac{\epsilon\left(\alpha+\beta \gamma t_{0}\right)^{2}-\alpha}{\left(\gamma t_{0}\right)^{2}}-[a(t)]^{\epsilon} \frac{\epsilon(\alpha+\beta t)^{2}-\alpha}{t^{2}}\right]$

Here $\epsilon=3+k, a(t)=a_{0}\left(t / t_{0}\right)^{\alpha} \operatorname{Exp}\left[\beta\left(t-t_{0}\right)\right]$ and

$a\left(\gamma t_{0}\right)=a_{0} \gamma^{\alpha} \operatorname{Exp}\left[\beta t_{0}(\gamma-1)\right]$, following equation (16).

A measure of the matter content of the universe, i.e. $M(t)=\rho a^{3}$, in this case is given by,

$M(t)=(\epsilon-1) \varphi_{0}[a(t)]^{\epsilon} \frac{\epsilon(\alpha+\beta t)^{2}-\alpha}{t^{2}}$

A measure of the dark energy content of the universe, i.e. $M_{d}(t)=\rho_{d} a^{3}$, is given by,

$M_{d}(t)=(\epsilon-1) \varphi_{0}\left[\left[a\left(\gamma t_{0}\right)\right]^{\epsilon} \frac{\epsilon\left(\alpha+\beta \gamma t_{0}\right)^{2}-\alpha}{\left(\gamma t_{0}\right)^{2}}-[a(t)]^{\epsilon} \frac{\epsilon(\alpha+\beta t)^{2}-\alpha}{t^{2}}\right]$

For the equations (30A, B), $\epsilon=3+k, a(t)=a_{0}\left(t / t_{0}\right)^{\alpha} \operatorname{Exp}\left[\beta\left(t-t_{0}\right)\right]$ and $a\left(\gamma t_{0}\right)=a_{0} \gamma^{\alpha} \operatorname{Exp}\left[\beta t_{0}(\gamma-1)\right]$, following equation (16).

This matter content of universe is found to decrease with time, due to the conversion of matter into dark energy. As a consequence of that, the dark energy content of the universe increases. Their sum $\left(M+M_{d}\right)$ is a conserved quantity. The density of all matter and dark energy is therefore given by,

$\rho_{\text {total }}(t)=\rho(t)+\rho_{d}(t)=\frac{M+M_{d}}{[a(t)]^{3}}=\frac{(2+k) \varphi_{0}}{[a(t)]^{3}}\left[a\left(\gamma t_{0}\right)\right]^{\epsilon} \frac{\epsilon\left(\alpha+\beta \gamma t_{0}\right)^{2}-\alpha}{\left(\gamma t_{0}\right)^{2}}$

The matter content of the universe is regarded as conserved in several studies $[20,21]$. We can express this conserved quantity as $M_{C}=\rho_{c}(t) a^{3}=\rho_{0} a_{0}{ }^{3}$. Thus, considering the matter content of the universe to be conserved, the density of matter $\left(\rho_{c}\right)$ can be expressed as,

$\rho_{c}(t)=\rho_{0}\left[\frac{a(t)}{a_{0}}\right]^{-3}$

Combining (28B) with (32) we get,

$\frac{\rho(t)}{\rho_{c}(t)}=\frac{1}{\rho_{0}}\left[\frac{a(t)}{a_{0}}\right]^{3+k}(2+k) \varphi_{0} \frac{(3+k)(\alpha+\beta t)^{2}-\alpha}{t^{2}}$

Equation (33) serves as a relation between $\rho(t)$ and $\rho_{c}(t)$, which enables one to compare the time dependence of their values. Substituting equation (18) into equation (14) one gets the following time dependent expression of the Brans-Dicke parameter $(\omega)$. 
$\omega(t)=2\left[\frac{\alpha(k+2)}{k^{2}(\alpha+\beta t)^{2}}-1-\frac{2}{k}-\frac{3}{k^{2}}\right]$

Combining the equations (16) and (28A) with equation (12) we get,

$R_{m}=\frac{e^{\beta(3+k)\left(t-\gamma t_{0}\right)} \gamma^{2-\alpha(3+k)}\left[(3+k)(\alpha+\beta t)^{2}-\alpha\right]\left(\frac{t}{t_{0}}\right)^{\alpha(3+k)} t_{0}{ }^{2}}{t^{2}\left[(3+k)\left(\alpha+\beta \gamma t_{0}\right)^{2}-\alpha\right]}$

Combining the equations (16) and (28A) with equation (13) we get,

$R_{d}=1-\frac{e^{\beta(3+k)\left(t-\gamma t_{0}\right)} \gamma^{2-\alpha(3+k)}\left[(3+k)(\alpha+\beta t)^{2}-\alpha\right]\left(\frac{t}{t_{0}}\right)^{\alpha(3+k)} t_{0}{ }^{2}}{t^{2}\left[(3+k)\left(\alpha+\beta \gamma t_{0}\right)^{2}-\alpha\right]}$

The above pair of equations $(35,36)$ enable one to study the time dependence of the parameters $R_{m}$ and $R_{d}$ for $k=k_{-}$and for different values of the parameter $\gamma$ where $\gamma<1$.

\section{Graphical Analysis of Theoretical Findings}

Figure 1 shows a plot of the scale factor and Hubble parameter as functions of time. They are found to increase and decrease with time respectively, as obtained from other studies. The constants of the scale factor (equation 16) have been so adjusted that we get the experimentally obtained value of Hubble parameter at the present time, i.e., $t=t_{0}$.

Figure 2 has a plot of the deceleration parameter and the Brans-Dicke scalar field parameter as functions of time. As time goes on, the logarithm of scalar field $(\varphi)$ shows a steady fall. The deceleration parameter $(q)$ is found to decrease with time with a gradually decreasing rate. Our scale factor (equation 16) has been chosen empirically in a manner such that the deceleration parameter changes sign as a function of time. This is evident from the graph. The constants of the scale factor have been so adjusted that we get the experimentally obtained value of $q$ at the present time, i.e., $t=t_{0}$. Over a long range of time $\left(t=0.5 t_{0}\right.$ onwards) the deceleration parameter changes very little, becoming more negative with time very slowly.

Figure 3 shows variations of densities of matter and dark energy of the universe as functions of time. These are based on the equations (28A, B) and (29), with $\rho_{m}=\rho(t)$. Here we have taken $\gamma=0.5$, implying that the matter to dark energy conversion began at $t=0.5 t_{0}$. At this time the dark energy density rises from zero and, after reaching a peak value, it decreases with time. The density of matter decreases with time monotonically, partly due to expansion of universe and partly due to its conversion into dark energy.

Figure 4 shows a plot of deceleration parameter $(q)$ and the Brans-Dicke parameter $(\omega)$ as functions of the scalar field $(\varphi)$. In Figure 2 we have seen that the scalar field decreases with time. As $\varphi$ decreases, $q$ changes its value from positive to negative, decreasing much more slowly in its negative region, in comparison with its positive region. The value of $\omega(\varphi)$ is negative over the entire range of study, having almost a constant value over a range of $\varphi$ from nearly $10^{9}$ to $10^{11}$. It is evident from the Figures 2 and 4 that, during the accelerated state of expansion of the universe, the values of $\omega(\varphi)$ and $q$ show a much slower change compared to the phase of deceleration.

Figure 5 shows a plot of relative proportions of matter and dark energy, $R_{m}$ and $R_{d}$ respectively, as functions of time. Matter to energy conversion began at $t=\gamma t_{0}$. It is evident from these curves, that for $\gamma=$ 0.8 , their values are close to experimental observations $\left[R_{m} \sim 0.3, R_{d} \sim 0.7\right]$ at the present epoch $\left(t=t_{0}\right)$, as obtained from other studies $[23,26]$.

Figure 6 shows a plot of $G / G_{0}$ and $\dot{G} / G$ as functions of time. The gravitational constant increases with time at a gradually increasing rate. This is consistent with other studies [24]. The value of $\dot{G} / G$ decreases with time with a rate which also decreases with time. Comparing Figure 6 with Figure 2, it can be said that $\dot{G} / G$ changes very little after the beginning of the phase of accelerated expansion.

Figure 7 shows a plot of deceleration parameter $(q)$ and Brans-Dicke parameter $(\omega)$ as functions of matter density $\left[\rho_{m} \equiv \rho(t)\right]$. The density of matter decreases with time (Figure 3). As $\rho_{m}$ decreases, the dark energy content of the universe increases, causing $q$ to decrease from a positive to a negative value. Due to the expansion of the universe, the dark energy density also decreases, as shown in Figure 3, causing a reduction in the rate of change of $q$. The value of $\omega$ remains close to $-3 / 2$ over this range of $\rho_{m}$, becoming less negative as $\rho_{m}$ decreases.

Figure 8 shows the variations of the $\operatorname{ratios} G / G_{0}$ and $\dot{G} / G$ as functions of matter density $\left[\rho_{m} \equiv \rho(t)\right]$. As $\rho_{m}$ decreases, the dark energy content of the universe increases, causing $G$ to increase [24]. As $\rho_{d}$ decreases 
with time, as shown in Figure 3, the rate of increase of $G$ gets reduced. As $\rho_{m}$ decreases, $\dot{G} / G$ is found to decrease, its value remaining within the well accepted upper limit of $4 \times 10^{-10} \mathrm{Yr}^{-1}$ [25].

All these observations lead to an insight into the nature of changes of the cosmological parameters on account of the changes of the matter and the dark energy contents, their respective densities and also their relative proportions in the universe.

\section{Conclusion}

A theoretical model has been developed here, regarding the time dependence of various cosmological parameters that characterize and govern the present accelerated expansion of the universe. The parameter $k$ in the empirical expression of the scalar field, determines the speed at which the scalar field changes as a function of scale factor and time. Two values of $k$, obtained as two roots of a quadratic equation, are found to be negative. Therefore, the reciprocal of scalar field, i.e. the gravitational constant increases with time. It is evident from the figures that the time dependence of the deceleration parameter, dark energy density, scalar field and Brans-Dicke parameter have some correlation among them. It appears that the Brans-Dicke parameter shows a change of phase along with the change of sign of the deceleration parameter, which indicates a transition from a phase of deceleration to acceleration of the expanding universe. Both $\omega$ and $q$ are found to reach a saturation value as time progresses. The parameter $k$ also governs the rate of change of the matter density and gravitational constant with time. To get a better theoretical model, there must be a provision in the empirical expression of $\varphi$, to vary the value of $k$. Instead of choosing this parameter to be a constant, one may replace it by a slowly varying function of time with constant parameters, which are to be determined from various boundary conditions. By using this provision of $k$ variation, one may get a better insight into the mechanism that governs the conversion of matter into dark energy and also the role played by the Brans-Dicke parameter. As part of a future plan of study, one may also choose a different empirical form of the scale factor to determine the time dependent functional form different cosmological parameters that have been dealt with in the present study. Without taking recourse to any specific mechanism of interaction between matter and the scalar field, the present model explores the time dependence of various cosmological factors connected to cosmic expansion, on the basis of a simple assumption regarding the conversion of matter into dark energy. The present model has also given us a glimpse of how the inter-conversion between matter and dark energy controls the depletion of matter content of the universe and its effect upon the time dependence of various parameters characterizing the expansion of the universe.

\section{FIGURES}

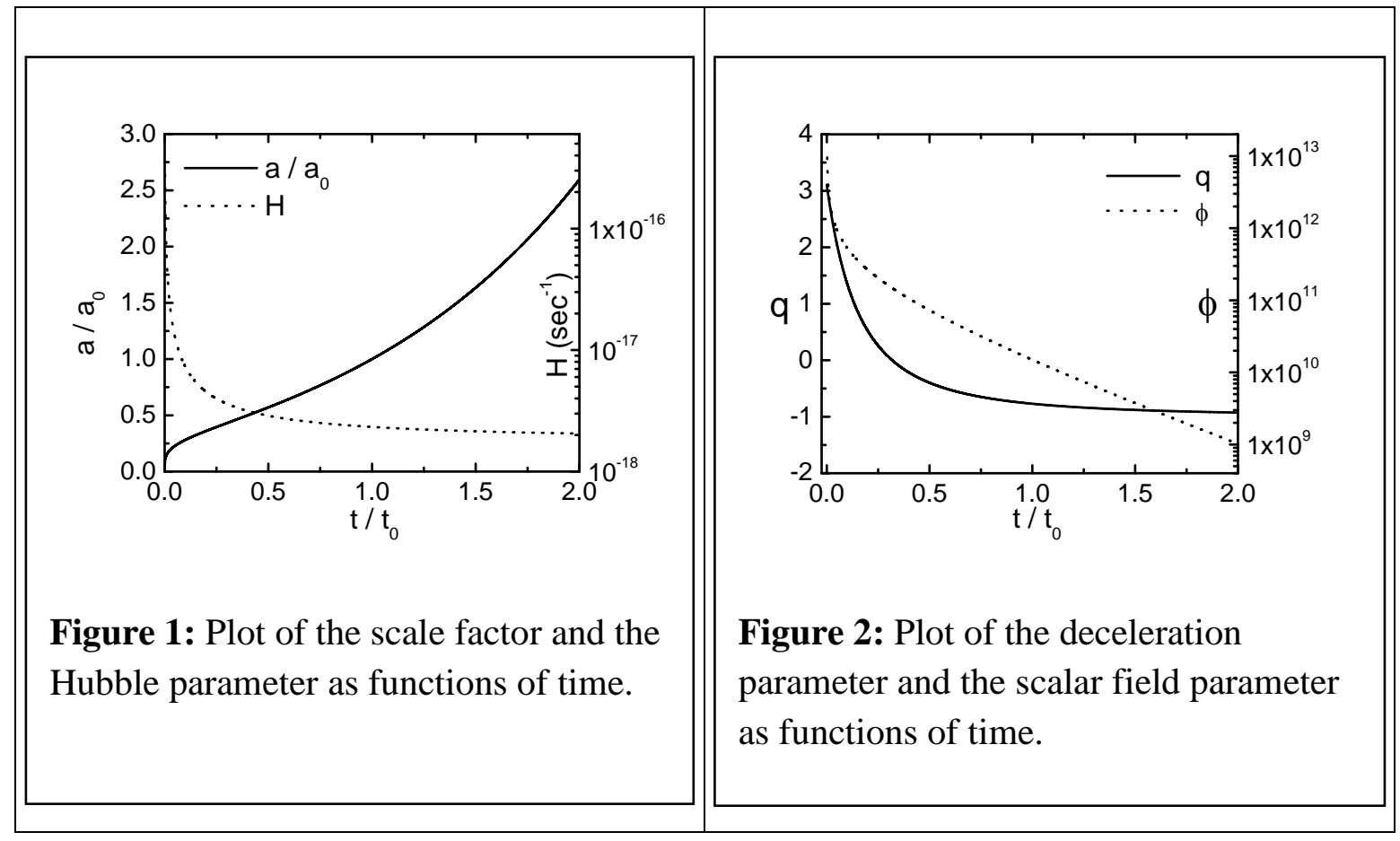




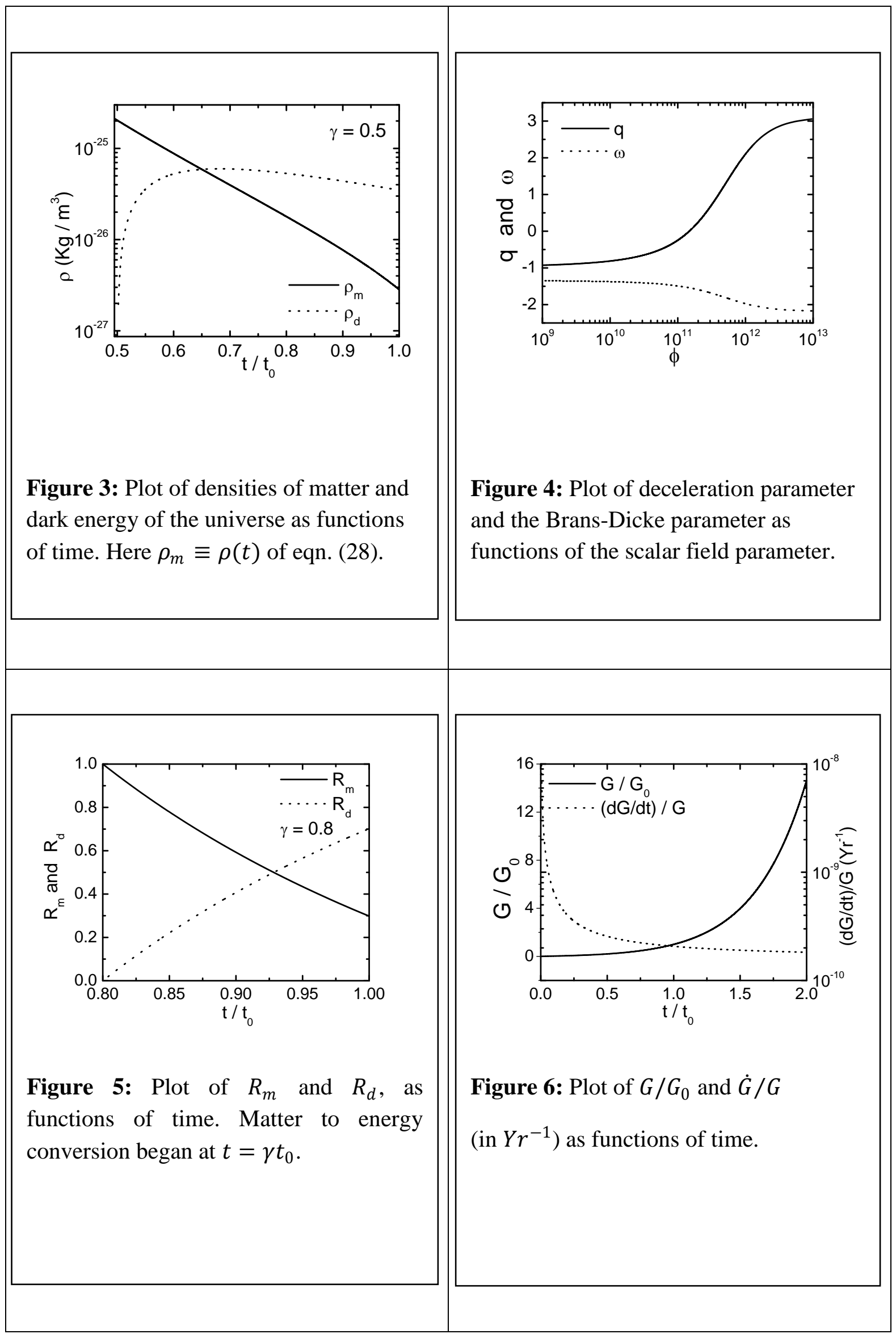




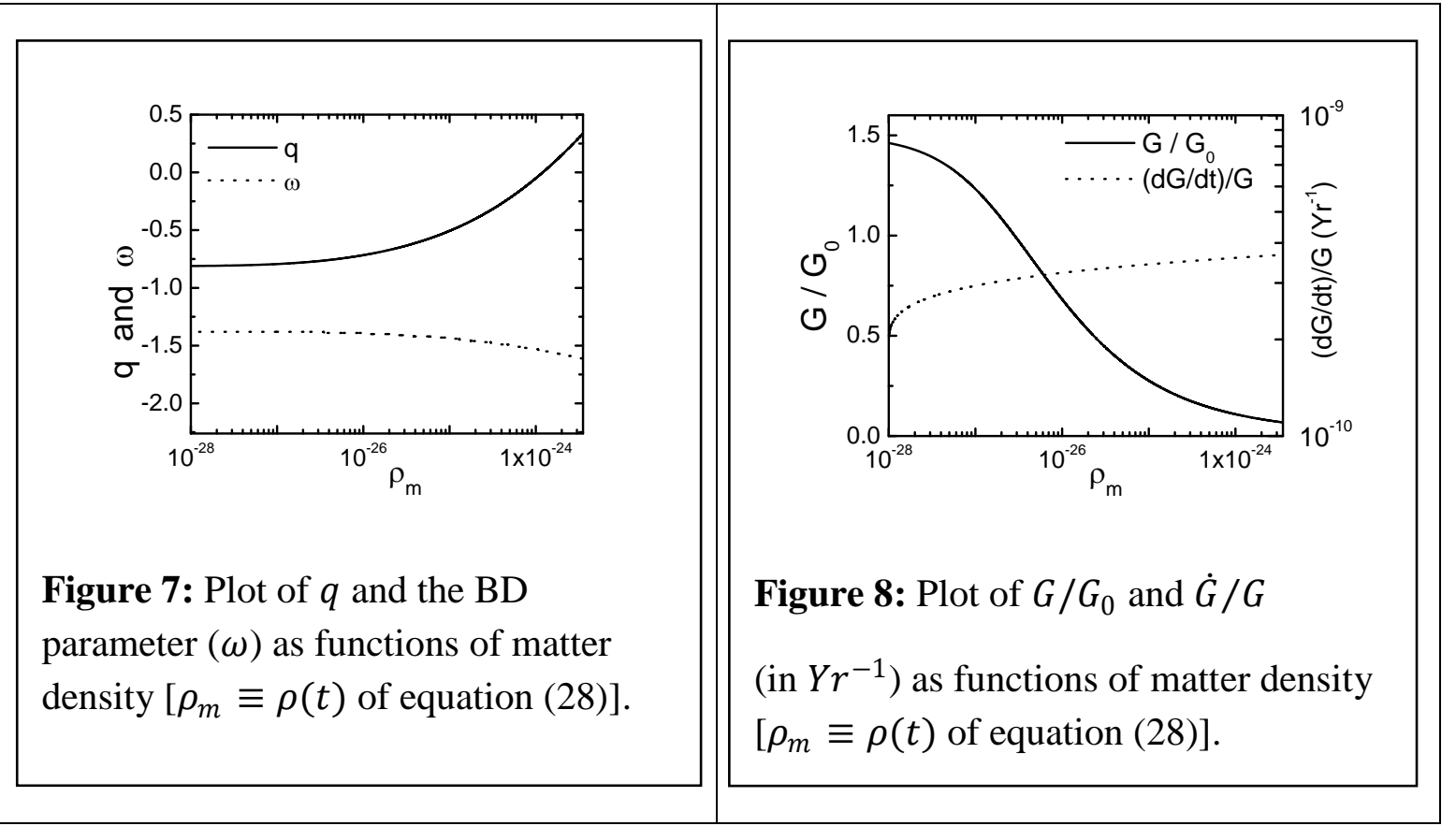

\section{References}

[1]. S. Perlmutter et al., Measurements of Omega and Lambda from 42 High-Redshift Supernovae, The Astrophysical Journal, 517, 1999, 565-586.

[2]. A. G. Reiss et al., Type Ia Supernova Discoveries at z>1 From the Hubble Space Telescope: Evidence for Past Deceleration and Constraints on Dark Energy Evolution, The Astrophysical Journal, 607, 2004, 665-687.

[3]. T. Padmanabhan, Cosmological Constant-the weight of the vacuum, Physics Reports, 380, 2003, 235-320.

[4]. V. Sahni, The Cosmological Constant Problem and Quintessence, Classical and Quantum Gravity, 19, $2002,3435-3448$.

[5]. R. G. Vishwakarma, A Machian Model of Dark Energy, Classical and Quantum Gravity, 19, 2002, 4747-4752.

[6]. A.A. Sen and S. Sethi, Quintessence model with double exponential potential, Physics Letters B, 532 (3), $2002,159-165$.

[7]. T. Padmanabhan, Accelerated expansion of the universe driven by tachyonic matter, Physical Review D, 66, 2002, 021301.

[8]. C. Armendariz-Picon, V. Mukhanov, and P. J. Steinhardt, Dynamical solution to the problem of a small cosmological constant and late-time cosmic acceleration, Physical Review Letters, 85 (21), 2000, 4438-4441.

[9]. C. Gao, F. Wu, X. Chen and Y.G. Shen, A Holographic Dark Energy Model from Ricci Scalar Curvaturear, Xiv:0712.1394V4 [astro-ph], 2008, 1-7.

[10]. W. Zimdahl, D. Pavon, Interacting quintessence and the coincidence problem, arXiv:astro-ph/0404122v1, $2004,1-3$.

[11]. N. Banerjee, S. Das and K. Ganguly, Chameleon field and the late time acceleration of the universe, Pramana-Journal of Physics, 74(3), 2010, L481-L489.

[12]. O. Bertolami and P. J. Martins, Nonminimal coupling and quintessence, Physical Review D, 61, 2000, 064007-064012.

[13]. A. Sheykhi, K. Karami, M. Jamil, E. Kazemi, M. Haddad, Power-Law Entropy-Corrected HDE and NADE in Brans-Dicke Cosmology, International Journal of Theoretical Physics, 51 (6), 2012, 1663-1673.

[14]. M. Jamil, I. Hussain and D. Momeni, Cosmic Evolution in Brans-Dicke Chameleon Cosmology, arXiv:1105.3893v2 [physics.genph], 2011, 1-15.

[15]. A. Pasqua and I. Khomenko, Interacting Ricci Logarithmic Entropy-Corrected Holographic Dark Energy in Brans-Dicke Cosmology, International Journal of Theoretical Physics, 52 (11), 2013, 3981-3993.

[16]. N. Banerjee and D. Pavon, A quintessence scalar field in Brans-Dicke theory, Classical and Quantum Gravity, 18, $2001,593-599$.

[17]. N. Banerjee and S. Das, A late time acceleration of the univberse with two scalar fields: many possibilities, Modern Physics Letters A, 21 (35), 2006, 2663-2670.

[18]. S. Das and N. Banerjee, An interacting scalar field and the recent cosmic acceleration, General Relativity and Gravitation, 38 (5), 2006, 785-794.

[19]. T. Clifton and J. D. Barrow, Decaying Gravity, arXiv:gr-qc/0603116v2, 2006, 1-13.

[20]. N. Banerjee and K. Ganguly, Generalised Scalar-Tensor theory and the cosmic acceleration, International Journal of modern Physics D, 18 (3), 2009, 445-451.

[21]. S. Roy, S. Chattopadhyay and A. Pasqua, A study on the dependence of the dimensionless Brans-Dicke parameter on the scalar field and their time dependence, European Physical Journal Plus, 128 (147), 2013, 1-16.

[22]. C. Brans and R.H. Dicke, Mach's Principle and a Relativistic Theory of Gravitation, Physical Review, 124, 1961, 925-935.

[23]. S Das, A Al Mamon, An Interacting model of Dark Energy in Brans-Dicke theory, Astrophysics and Space Science, 351, 2014, 651660 .

[24]. A. Pradhan, B. Saha and V. Rikhvitsky, Bianchi type-I transit cosmological models with time dependent gravitational and cosmological constants: re-examined, Indian Journal of Physics, 89 (5), 2015, 503-513.

[25]. S. Weinberg, Gravitation and Cosmology: Principles and Applications of the General Theory of Relativity (John Wiley \& Sons (Asia) Pte. Ltd., Singapore: 630, 1972).

[26]. S. Roy, A Theoretical Study of the Cosmic Expansion in the Framework of Brans-Dicke Theory, IOSR Journal of Applied Physics (IOSR-JAP), 8(3) Version-III, 2016, 4-12. 